\title{
Dose-Dependent Risk Reduction for Myocardial Infarction with Eicosapentaenoic Acid: a Meta-analysis and Meta-regression Including the STRENGTH Trial
}

\author{
Philip Sarajlic $^{1,2} \cdot$ Gonzalo Artiach $^{1}$ · Susanna C. Larsson ${ }^{3,4} \cdot$ Magnus Bäck $^{1,5}$
}

Accepted: 28 May 2021 / Published online: 8 June 2021

(c) The Author(s) 2021

Numerous trials have investigated the role of the long-chain omega-3 polyunsaturated fatty acids (PUFAs) eicosapentaenoic acid (EPA) and docosahexaenoic acid (DHA) for the prevention of cardiovascular events, with renewed interest sparked by recent findings that omega-3 PUFAs are substrates for lipid mediators of the resolution of inflammation $[1,2]$. Whereas some meta-analyses indicated no risk reduction for MI by omega-3 PUFA [3], the most recent revealed a significantly $8 \%$ lower risk of MI, with higher doses of omega-3 PUFA conferring greatest protection [4]. Since then, the results of the STRENGTH trial were reported and showed that high-dose omega-3 PUFA ( $4 \mathrm{~g}$, of which $2.2 \mathrm{~g}$ EPA and $0.8 \mathrm{~g}$ DHA) supplementation had no significant effects on either the composite primary endpoint or non-fatal MI [5]. Therefore, we aimed to update existing meta-analyses $[3,4]$ with subsequently published trials to determine the association between EPA and DHA and their dosages with MI risk. We included all trials from the meta-analysis by Aung et al. [3] along with five subsequently published trials, namely, REDUCE-IT, VITAL, ASCEND (references in [6]), OMEMI [7], and STRENGTH [5]. The study pool consisted of randomized trials with minimally

Philip Sarajlic

philip.sarajlic@ki.se

1 Department of Medicine Solna, Karolinska Institutet, Stockholm, Sweden

2 Department of Medicine, Translational Cardiology, Karolinska University Hospital, Hälsovägen 7C, 14157 Stockholm, Sweden

3 Unit of Cardiovascular and Nutritional Epidemiology, Institute of Environmental Medicine, Karolinska Institutet, Stockholm, Sweden

4 Department of Surgical Sciences, Uppsala University, Uppsala, Sweden

5 Department of Cardiology Huddinge, Karolinska University Hospital, Stockholm, Sweden
500 patients and a follow-up period of at least one year that analyzed the association between omega-3 PUFA supplementation and vascular events. In total, 15 relevant trials were included. While both fatal and non-fatal MI outcomes were analyzed, in this report, we present analyses on nonfatal MI risk since more non-fatal MI events were recorded in the included trials, making this approach more statistically powerful. For each trial, Peto odds ratio was calculated to determine effect sizes. A meta-analytic scatterplot was created to visualize the risk of MI in each trial based on the dosage for EPA and DHA, respectively, using a randomeffects model. A meta-analytic regression line was fitted in the scatterplot to determine the risk trend and slope for the two omega-3 PUFAs. All statistical calculations were done using the suite of commands, "meta," in Stata version 16 (StataCorp. 2019. Release 16. College Station, TX: StataCorp LLC). A two-sided alpha value of 0.05 was used to determine statistical significance. Despite the non-significant effects of the latest trial [5], omega-3 supplementation was associated with a statistically significant lower odds of nonfatal MI (odds ratio 0.91; 95\% CI 0.83-0.99) in the metaanalysis of 15 studies, with moderate heterogeneity between estimates from individual trials $\left(\mathrm{I}^{2}=44 \%\right)$ (Fig. 1A). A significant dose-dependent risk reduction of non-fatal MI was observed for EPA (Fig. 1B). While DHA was significantly associated with a lower risk of non-fatal MI at low doses, the risk reduction lost significance at higher doses (Fig. 1B). In a bivariate meta-regression analysis, with EPA and DHA as covariates, EPA achieved a significant non-fatal MI risk reduction $(P=0.048 ; \mathrm{z}=-1.97)$, while the effect of DHA was non-significant $(P=0.477 ; \mathrm{z}=0.71)$. In a sensitivity analysis including only double-blind trials, a univariate meta-regression revealed significant $(P=0.048 ; \mathrm{z}=-1.98)$ beneficial risk reduction properties of EPA. Together, these findings point to a dose-dependent risk reduction of non-fatal MI with increasing EPA dosage, regardless of DHA intake. In order to account for these differential effects, one could look at 


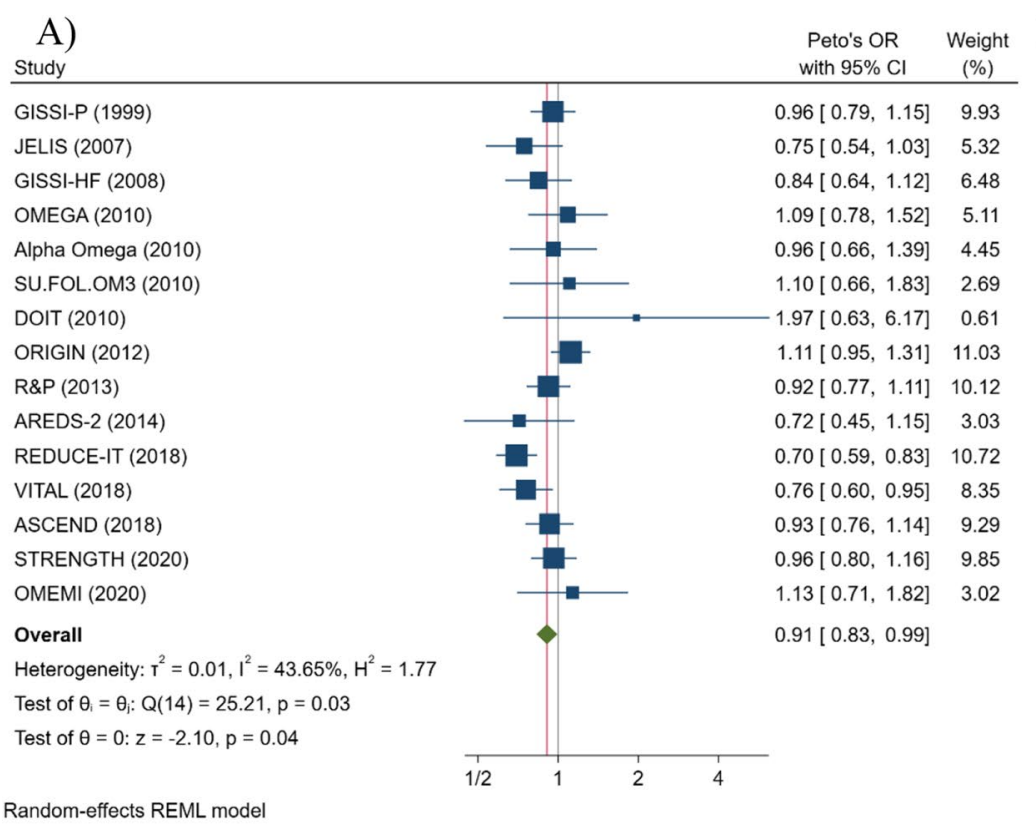

B)

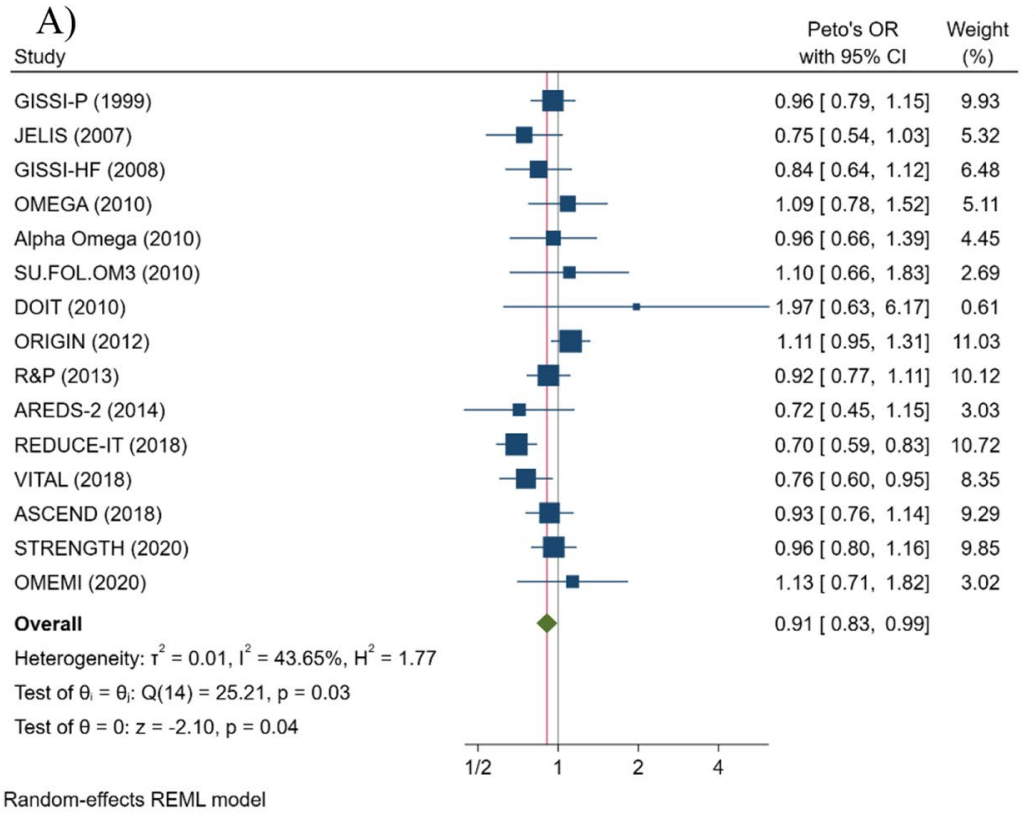

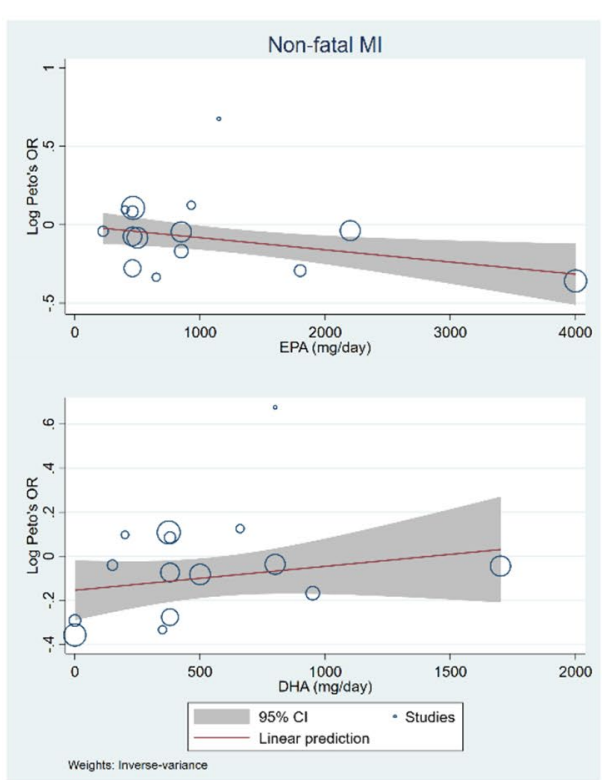

Fig. 1 Meta-analysis (A) and meta-regression (B) with 15 trials illustrating the relationship between omega-3 PUFAs and non-fatal MI risk. (B) shows log Peto odds ratios on the y-axis and the omega-3

atherosclerosis and its pathophysiology. In addition to their anti-thrombotic, triglyceride-lowering, and atherogenic remnant particle lowering effects, EPA and DHA serve as substrates for specialized pro-resolving mediators (SPMs) ${ }^{2}$, which promote the resolution of atherosclerotic inflammation [1]. Preclinical atherosclerosis models indicate that EPA leads to the formation of SPMs capable of tipping the cardiovascular homeostatic balance towards inflammation resolution [8]. A limitation of our meta-analysis is the presence of variances in disease severity across different study populations, potentially contributing to heterogeneity between the trials. Furthermore, analyses on fatal MI were not feasible due to a lack of reported outcome data in the included trials. In conclusion, this contemporary meta-analysis showed that EPA was associated with a significant risk reduction of non-fatal MI in a dose-dependent fashion. The association persisted in a model adjusting for DHA intake, emphasizing the role of EPA supplementation in CHD prevention. Further studies on EPA downstream metabolites are warranted.
PUFA dose on the $\mathrm{x}$-axis. Each circle in the scatterplot represents one study, and its area is proportional to the inverse of the standard error

\section{Declarations}

Conflict of Interest The authors declare no competing interests.

Open Access This article is licensed under a Creative Commons Attribution 4.0 International License, which permits use, sharing, adaptation, distribution and reproduction in any medium or format, as long as you give appropriate credit to the original author(s) and the source, provide a link to the Creative Commons licence, and indicate if changes were made. The images or other third party material in this article are included in the article's Creative Commons licence, unless indicated otherwise in a credit line to the material. If material is not included in the article's Creative Commons licence and your intended use is not permitted by statutory regulation or exceeds the permitted use, you will need to obtain permission directly from the copyright holder. To view a copy of this licence, visit http://creativecommons.org/licenses/by/4.0/.

\section{References}

1. Bäck M, Yurdagul A Jr, Tabas I, Oorni K, Kovanen PT. Inflammation and its resolution in atherosclerosis: mediators and therapeutic opportunities. Nat Rev Cardiol. 2019;16(7):389-406.

2. Serhan CN. Pro-resolving lipid mediators are leads for resolution physiology. Nature. 2014;510(7503):92-101.

3. Aung T, Halsey J, Kromhout D, Gerstein HC, Marchioli R, Tavazzi L, Geleijnse JM, Rauch B, Ness A, Galan P, Chew EY, Bosch J, Collins R, Lewington S, Armitage J, Clarke R. 
Associations of omega-3 fatty acid supplement use with cardiovascular disease risks: meta-analysis of 10 trials involving 77917 individuals. JAMA Cardiol. 2018;3(3):225-34.

4. Hu Y, Hu FB, Manson JE. Marine omega-3 supplementation and cardiovascular disease: an updated meta-analysis of 13 randomized controlled trials involving 127477 participants. J Am Heart Assoc. 2019;8(19):e013543.

5. Nicholls SJ, Lincoff AM, Garcia M, Bash D, Ballantyne CM, Barter PJ, Davidson MH, Kastelein JJP, Koenig W, McGuire DK, Mozaffarian D, Ridker PM, Ray KK, Katona BG, Himmelmann A, Loss LE, Rensfeldt M, Lundström T, Agrawal R, Menon V, Wolski K, Nissen SE. Effect of high-dose omega-3 fatty acids vs corn oil on major adverse cardiovascular events in patients at high cardiovascular risk: the STRENGTH randomized clinical trial. JAMA. 2020;324(22):2268-80.

6. Bäck M, Hansson GK. Omega-3 fatty acids, cardiovascular risk, and the resolution of inflammation. FASEB J. 2019;33(2):1536-9.
7. Kalstad Are A, MyhrePeder L, Laake K, TveitSjur H, Schmidt Erik B, Smith P, Nilsen Dennis Winston T, Tveit A, Fagerland Morten W, Solheim S, Seljeflot I, Arnesen H. Effects of n-3 fatty acid supplements in elderly patients after myocardial infarction: a randomized controlled trial. Circulation. 2021;143:528-39.

8. Laguna-Fernandez A, Checa A, Carracedo M, Artiach G, Petri MH, Baumgartner R, et al. ERV1/ChemR23 signaling protects against atherosclerosis by modifying oxidized low-density lipoprotein uptake and phagocytosis in macrophages. Circulation. 2018;138(16):1693-705.

Publisher's Note Springer Nature remains neutral with regard to jurisdictional claims in published maps and institutional affiliations. 